Technological University Dublin

DÜBLIN

ARROW@TU Dublin

Articles

DIT Biophotonics and Imaging

2012

\title{
Analysis of Human Skin Tissue by Raman Microspectroscopy: Dealing with the Background
}

\author{
Franck Bonnier \\ Technological University Dublin, Franck.Bonnier@tudublin.ie \\ Syed Mehmood Ali \\ Technological University Dublin \\ Peter Knief \\ Technological University Dublin
}

See next page for additional authors

Follow this and additional works at: https://arrow.tudublin.ie/biophonart

Part of the Diagnosis Commons, and the Physics Commons

\section{Recommended Citation}

Bonnier, F. et al (2012) Analysis of human skin tissue by Raman microspectroscopy: Dealing with the background, Vibrational Spectroscopy, 61, pp.124-132. http://dx.doi.org/10.1016/j.vibspec.2012.03.009

This Article is brought to you for free and open access by the DIT Biophotonics and Imaging at ARROW@TU Dublin. It has been accepted for inclusion in Articles by an authorized administrator of ARROW@TU Dublin. For more information, please contact arrow.admin@tudublin.ie, aisling.coyne@tudublin.ie,gerard.connolly@tudublin.ie. Funder: HEA PRTLI Cycle 4 NBIPI

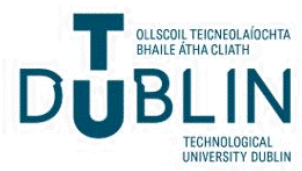




\section{Authors}

Franck Bonnier, Syed Mehmood Ali, Peter Knief, Helen Lambkin, Kathleen Flynn, Vincent McDonagh, Claragh Healy, Thomas Lee, Fiona Lyng, and Hugh J. Byrne 


\title{
Analysis of human skin tissue by Raman microspectroscopy: Dealing with the background
}

\author{
F. Bonnier ${ }^{1}$, S.M. Ali ${ }^{1}$, P. Knief ${ }^{1}$, H. Lambkin ${ }^{2}$, K. Flynn ${ }^{2}$, V. McDonagh ${ }^{3}$, C. Healy ${ }^{3}$, \\ T.C. Lee ${ }^{3}$, F.M. Lyng ${ }^{1}$, H.J. Byrne ${ }^{1}$ \\ ${ }^{1}$ Focas Research Institute, Dublin Institute of Technology, Kevin Street, Dublin 8, \\ Ireland \\ ${ }^{2}$ School of Biological Sciences, Dublin Institute of Technology, Kevin Street, Dublin 8, \\ Ireland. \\ ${ }^{3}$ Department of Anatomy, Royal College of Surgeons in Ireland, 123 St. Stephen's \\ Green, Dublin 2, Ireland.
}

Vibrational Spectroscopy, 61, 124-132 (2012)

\begin{abstract}
:
Raman microspectroscopy is widely used for molecular characterisation of tissue samples. Nevertheless, when working in vitro on tissue sections, the presence of a broad background to the spectra remains problematic and its removal requires advanced methods for pre-processing of the data. To date, research efforts have been primarily devoted to development of techniques of statistical analysis to extract the relevant information contained in the spectra. However, few attempts have been made to understand the origin of the background and to improve the protocols used for the collection of Raman spectra that could lead to the reduction or elimination of the background. It has been demonstrated that measurement at $785 \mathrm{~nm}$ in water immersion significantly reduces the Raman background of both pure biochemical components and tissue sections, associating the background at $785 \mathrm{~nm}$ with a scattering phenomenon rather than fluorescence. It is however of interest to probe the dependence of the observed background and any time evolution normally associated with photobleaching of fluorophores, under dry and immersed conditions, on the source wavelength. Using $785 \mathrm{~nm}$ or $660 \mathrm{~nm}$ as source, extended exposure of dried skin tissue sections to the laser results in a time dependent reduction of the background present in the Raman spectra. When working in water immersion, the overall background as well as the evolution over time is greatly reduced and the background is seen to stabilise after 20 seconds exposure. Using $532 \mathrm{~nm}$ or $473 \mathrm{~nm}$ as source for the examination of dried tissue sections, visible photodamage of the sample limits the laser power usable for the collection of spectra to $5 \mathrm{~mW}$. Immersion of the tissue sections protects against photodamage and laser powers of up to $30 \mathrm{~mW}$ can be used without any visible damage. Under these conditions, the background is significantly reduced and good quality Raman spectra can be recorded. By adapting the protocol usually used for the collection of Raman spectra, this study clearly demonstrates that other approaches rather than mathematical manipulation of the data can be used to deal with the intrinsic background commonly observable. Notably, the dependence of the background and its time evolution under prolonged exposure on
\end{abstract}


sample environment potentially sheds light on its origin as due to sample morphology (scattering) rather than chemical content (fluorescence). Overall, the study demonstrates that, in addition to reduced background, the photostability of the samples is significantly enhanced in an immersion geometry.

Keywords: Raman Spectroscopy; human tissue analysis; Raman background; Fluorescence; Scattering; Photodamage

\footnotetext{
* Corresponding author.

Focas Research Institute, Dublin Institute of Technology, Dublin (Ireland).

Tel.: +3531 4027917; Fax: +3531 4027904;

E-mail address: fbonnier@dit.ie (F. Bonnier)
}

\section{Introduction}

The potential of Infrared and Raman spectroscopy as medical diagnostic tools has been well demonstrated [1-5]. The main advantages of these techniques are that they provide a non invasive, label free molecular fingerprint of tissue and cells. In the last few years, many further reaching applications have been described, including in radiobiology [6], toxicology [7] and pharmacokinetics [8]. Coupled with adapted multivariate analysis, the specificity of the information obtained can be used for the identification of different pathologies $[9,10]$, or variations in metabolism as a result of external agents $[11,12]$. As it commonly employs optical or near infrared sources, Raman spectroscopy exhibits a significantly high lateral resolution and enables access to subcellular information [13, 14]. For this reason, it is often used in parallel with FTIR spectroscopy to provide more detailed information about the tissues under investigation $[15,16]$.

Common to the development of both these techniques is the importance of the preprocessing of the data before progressing to more advanced analysis. The spectral 
background can be a combination of many signals contributing to and "contaminating" the spectra recorded. For example, water exhibits a strong absorption in the infrared range and samples have ideally to be dried before recording any spectra [17]. In Raman spectroscopy, however, due to its weak scattering efficiency, measurements realized in water immersion allow for example measurement of live cells $[18,19]$. The selection of the substrates is also important, as glass slides or Petri dishes are unsuitable for Raman spectroscopy, as the intrinsic material response overlaps and can swamp the tissue or cellular signals. Usually $\mathrm{CaF}_{2}$ or $\mathrm{ZnSe}$ windows are preferred as they are compatible with both techniques. Many improvements have already been achieved in the development of protocols for sample preparation in order to increase the quality of the data collected, but even using optimized optics, substrates and taking into consideration the different limits offered by the techniques, a substantial background can remain in the data collected. This background is a result of the interaction between the light used for the analysis and the samples and is due at least in part to the inherent physical inhomogeneity. In infrared spectra, the phenomenon has been attributed to Mie scattering and/or surface reflections, which can contribute a broad undulating baseline off resonance and a distortion of the spectral features on resonance [20,21]. Different methods for spectral correction have been recently proposed in order to remove this effect from the data sets [22].

Raman spectra can also be affected by the presence of a background making it difficult to reliably process the data for analysis. The origin is often attributed to fluorescence from the samples, due to the proximity of the source wavelength to the excitation bands of some biomolecular constituents of the cells or tissue $[23,24]$. It is important to note, however, that for a material to fluoresce, it must absorb at the irradiating wavelength. 
Fluorescence spectroscopy has been explored as a potential probe of malignancies in for example skin, the principle chromophores being nicotinamide adenine dinucleotide $(\mathrm{NADH})$, collagen, elastin, tryptophan, flavins, and porphyrins [25, 26]. However, excitation wavelengths are typically less than $400 \mathrm{~nm}$ and while some chromophores such as hemoglobin absorb at visible wavelengths, there is little or no fluorescence higher than $\sim 600 \mathrm{~nm}$. Notably, similar baselines are observable using source wavelengths as long as $785 \mathrm{~nm}$ or $830 \mathrm{~nm}[24,27]$. In skin, melanin provides the pigment and absorbs across the visible spectrum, fluorescing in the near infrared [28, 29]. However, melanin is confined to the basal layer of the epidermis and is not present in the dermis [30]. It is also notable that minimal backgrounds are observed in single cell spectroscopy at 785nm [13].

The phenomenon of large backgrounds to Raman spectra of proteins at wavelengths significantly longer than their absorption was reported as early as the 1970s [31]. The dependence on source wavelength pointed to a Raman like origin rather than fluorescence, and the background was also seen to "photo-bleach" under prolonged illumination at elevated laser powers, a process which became known as "drench and quench" [32]. More recently, in a study of pure proteins, the background has been demonstrated to be dependent on sample morphology rather than chemical content and so has been also associated with scattering of the source laser and the Raman bands themselves, causing them to enter the spectrometer in a noncollimated fashion, and thus spatially broaden on the grating and lose definition on the CCD, contributing to a broad background [33]. The process cannot be strictly considered as Mie scattering, however, which refers to elastic scattering from spherical objects of dimensions similar to the incident wavelength, and thus a more general term of scattering from the physically and 
chemically inhomogeneous materials may be more appropriate. As for the case of infrared spectroscopy, different methods have been proposed to remove this background from the data in order to ease the analysis $[34,35]$. However, although many studies focus on the pre-processing of the data and the development of new algorithms for removal of the background from the Raman data, few have addressed improvements of the protocols used for the spectral collection itself. In this regard, notably, it has been recently demonstrated that using a $785 \mathrm{~nm}$ laser source the background present in Raman spectra can be greatly reduced by using immersed rather than dried tissue sections [33]. To further demonstrate the importance of the pre-recording conditions underlying the presence of the background in Raman spectra and to further explore the origins of the background to the Raman spectrum, in this study we focus on the variation in spectral quality as a function of source wavelength $(473 \mathrm{~nm}, 532 \mathrm{~nm}, 660 \mathrm{~nm}$ and $785 \mathrm{~nm})$ and exposure time and source power. For this purpose, the dermal regions of fixed and subsequently dewaxed human skin sections have been examined with each laser line, in both dry and immersed conditions. Different exposure times have been used to compare the intensity of the background recorded and different approaches for the recording of the spectra will be discussed to further understand how the quality of the signal can be improved without any particular treatment of the tissue sections. This work further explores the origins of the Raman background, but ultimately demonstrates that other approaches rather than mathematical manipulation of the data can be employed to solve the issue of background present in the Raman spectra collected from formalin fixed paraffin processed and subsequently dewaxed human tissue sections, an invaluable source of tissue archives with associated patient histories. 


\section{Materials and Methods}

\subsection{Tissue section preparation}

Human skin tissues were obtained from the Anatomical Gift Programme from the Royal College of Surgeons in Ireland. All specimens were wax embedded as follows: (a) vacuum fixed in $10 \%$ buffered formal saline histograde, $\mathrm{pH}$ 6.8-7.2 (J.T.Baker; Deventer, The Netherlands) heated to $35^{\circ} \mathrm{C}$, (b) vacuum dehydration in industrial methylated spirits IMS T100 (Lennox; Dublin, Ireland) heated to $35^{\circ} \mathrm{C}$, (c) vacuum clearing in xylene (Serosep; Limerick, Ireland) heated to $35^{\circ} \mathrm{C}$, and (d) vacuum impregnation with Tissue-Tek III Embedding Wax with polymer added (Sakura Zoeterwoude, The Netherlands) and heated to $59^{\circ} \mathrm{C}$.

After embedding, the samples were sliced into $20 \mu \mathrm{m}$ sections using a microtome, and placed on $\mathrm{CaF}_{2}$ slides before being dried in an oven at $55^{\circ} \mathrm{C}$. The protocol for the dewaxing consists of immersion of the samples in a series of baths starting with two baths of xylene (BDH; Dorset, $\mathrm{UK}$ ) for $5 \mathrm{~min}$ and $4 \mathrm{~min}$, respectively, then two baths of Ethanol Absolut (Merck; Dorset, UK) for 3 min and 2 min, respectively, and finally a bath of Industrial Methylated Spirits 95\% (Lennox) for $1 \mathrm{~min}$.

\subsection{Raman spectroscopic measurements}

A Horiba Jobin-Yvon LabRAM HR800 spectrometer was used throughout this work. For the measurements, either a x100 objective (Olympus MPlanN) or a x100 immersion objective (Olympus LUMPlanF1) was employed, each providing a spatial resolution of $\sim 1 \mu \mathrm{m}$ at the sample. The confocal hole was set at $100 \mu \mathrm{m}$ for all measurements, the 
specified setting for confocal operation. Four different laser lines have been used for this study: $473 \mathrm{~nm}, 532 \mathrm{~nm}, 660 \mathrm{~nm}$ and $785 \mathrm{~nm}$ with source powers of $50 \mathrm{~mW}, 50 \mathrm{~mW}, 100$ $\mathrm{mW}$ and $300 \mathrm{~mW}$ respectively. After each change of laser line, the system was spectrally calibrated to the $520.7 \mathrm{~cm}^{-1}$ spectral line of silicon.

The LabRAM system is a confocal spectrometer that contains two interchangeable gratings (300 and 600 lines $/ \mathrm{mm}$ respectively). In the following experiments the 600 lines/mm grating was used for the $473 \mathrm{~nm}$ and $532 \mathrm{~nm}$ laser line, providing a spectral dispersion of approximately $1.5 \mathrm{~cm}^{-1} / 2 \mathrm{~cm}^{-1}$ per pixel. In order to maintain similar spectral resolution, the grating was changed to 300 for the $660 \mathrm{~nm}$ and $785 \mathrm{~nm}$ laser lines. The backscattered Raman signal was integrated for 10 seconds intervals over the spectral range from 400 to $1800 \mathrm{~cm}^{-1}$. The detector used was a 16-bit dynamic range Peltier cooled CCD detector. Images of the sample were acquired using a video camera within the system.

The samples were first recorded using the Olympus MPlanN objective in a dry state and then the same sections were immersed in distilled water before being recorded with the Olympus LUMPlanF1 objective. The Numerical Apertures and magnification of the respective objectives are defined for the specific modes in which they are applied. The Olympus MPlanN x100 objective has NA $=0.9$ while the Olympus LUMPlanF1 x100 water immersion objective has NA $=1$ in water. Thus, the voxel sizes can be considered approximately equal. Laser powers at the sample will differ only slightly because of different reflections at the tissue/air or water interface. All the spectra from the skin samples were recorded from the dermis, particularly rich in collagen, but critically devoid of melanin [30]. This location was selected in order to reduce as much as possible the 
variability due to differences in the biochemical composition between the different samples and ease the interpretation of the effect of the different laser lines used on the data recorded. In order to make a direct comparison of the stability of the Raman spectra and backgrounds under prolonged irradiation in dry and immersed state, a previously unirradiated spot was chosen for each measurement. Measurements have been performed on 6 tissue slides prepared from human skin coming from the hand. However, similar observations have been made on additional slides prepared from skin coming from the thigh.

Typically, Raman spectra are the average of $n$ accumulations contributing to the reduction of the noise contained in the data set recorded. The Raman intensity is directly proportional to the accumulation time. Therefore, the longer the acquisition, the higher the intensity of the signal obtained and thus the better the signal to noise ratio is. Although single point measurements have been performed throughout this work, acquisition times consistent with those commonly used for mapping have been chosen. When scanning a large skin area with a step size of $1 \mu \mathrm{m}$ or less, 10 seconds is the longest accumulations that could be used in order to achieve the mapping in a realistic time. As this work focuses on the presence of a background in the spectra recorded, only 1 accumulation has been performed for each acquisition, regardless of the noise present in the data.

\section{Results and discussion}

3.1 Presence of a background in the spectra recorded using the $785 \mathrm{~nm}$ wavelength 
Lateral resolution, depth penetration, photo-damage, prevalence of the background in the spectra, are some of the considerations which govern the choice of the wavelength to be used for the recording of Raman spectra from biological samples. The $785 \mathrm{~nm}$ laser line has become popular for investigations related to biomedical applications, from the study of the subcellular organisation in single cells to the diagnosis of pathological tissues $[13$, $14,36]$. It is notably widely used for live cell analysis, because of the absence of any obvious photo-degradation at this wavelength [37-39]. The background has been documented at irradiation wavelengths of $785 \mathrm{~nm}$ and even $830 \mathrm{~nm}$ [24], but is commonly assumed to be fluorescence and this assumption has entered the biomedical spectroscopy literature [23]. However, as shown in Figure 1, the background is significantly reduced when the tissue section is measured in immersion (figure 1B) rather than the dry state (figure 1A), and the Raman features of the tissue are significantly enhanced. For example, for the same illumination conditions, the amide 1 band $\left(\sim 1670 \mathrm{~cm}^{-1}\right)$ of the first acquired spectrum of the dry tissue has a peak height above background of $\sim 500$ counts, whereas, in immersion, the same feature has a peak height above background of $\sim 1000$ counts. Thus, the background has been proposed to have origin, at least in part, in scattering of the source and Raman lines, which can enter the spectrometer as stray light [33]. True fluorescence contributions to the background may be present at shorter wavelengths, however, as various biochemical components come into resonance. Resonance can also lead to photochemical degradation, however, and thus it is important to compare the scattering profiles of dry and immersed tissue as a function of Raman source wavelength. 


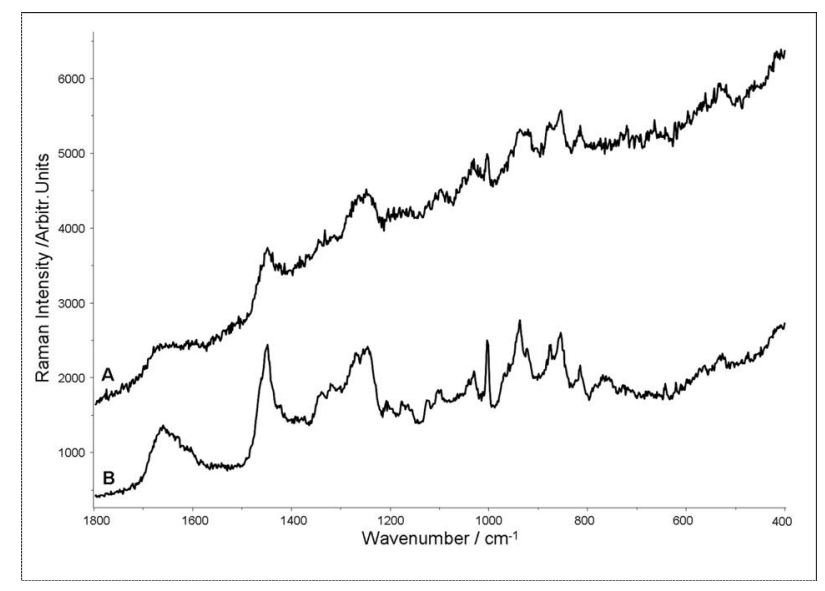

Fig.1: Examples of Raman spectra collected from human skin sections dry (A) or immersed in water (b) using a $785 \mathrm{~nm}$ wavelength. Spectra result from $1 \times 10 \mathrm{~s}$ acquisitions. No pre-processing or offset have been applied to the spectra.

In this study, measurements have been performed on skin sections deposited on $\mathrm{CaF}_{2}$ substrates using the 100x MPlanN objective. The tissue sections were dewaxed as described in the Materials and Methods section and kept in a desiccator until recorded. They are therefore considered to be in a dry state. A series of 10 s acquisitions was performed successively on the same spot in order to monitor the Raman spectral profiles over time. Figure $2 \mathrm{~A}$ presents a data set obtained with a laser intensity of $30 \mathrm{~mW}$ at the objective. An intense background is observed which makes the identification of specific features present in the spectra difficult. However, the level of the background evolves over time and prolonged exposure of the tissue to the laser results in a diminution of the intensity of the signal collected as indicated by the arrow in figure $2 \mathrm{~A}$. Thus, the duration of exposure to the laser source has an impact on the quality of the data collected, improving the signal to background ratio. Notably, no physical degradation of the sample is visibly discernible after prolonged exposure. 

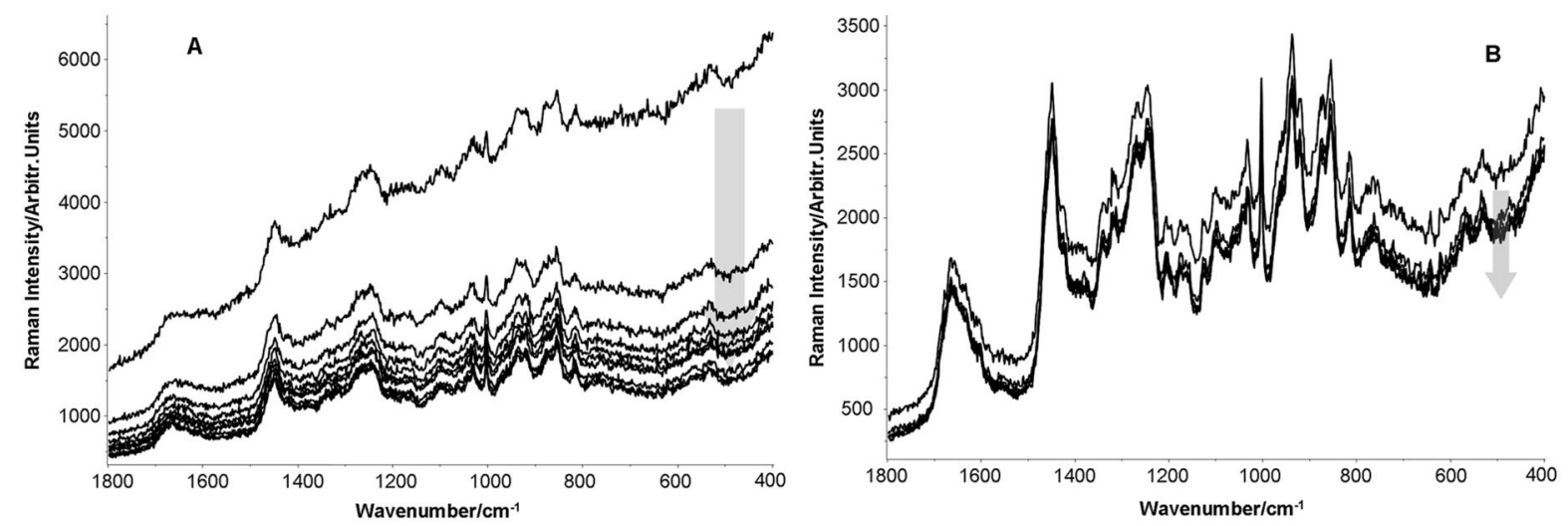

Fig.2: Raman spectra recorded from human skin sections placed on $\mathrm{CaF}_{2}$ windows. All spectra have been recorded using the $785 \mathrm{~nm}$ wavelength delivering $30 \mathrm{~mW}$ at the objective with an acquisition time of 10 s. Repeated measurements have been performed every 10s on dry (A) and immersed (B) skin. No pre-processing has been performed and no offset has been added to the plot.

The evolution of the background is better visualised by plotting the value at a single frequency, away from any of the tissue spectral features $\left(\sim 1750 \mathrm{~cm}^{-1}\right)$ as a function of time, as shown in figure 3. A rapid evolution over the first $\sim 30$ seconds is followed by a slower decay which persists even after the 10th acquisition of 10 seconds and the profile of the evolution is best fitted with a double exponential function of the form: $I_{B}=$ $A \exp \left(k_{1} t\right)+B \exp \left(k_{2} t\right)$, where $I_{B}$ is the background intensity, $A$ and $B$ are constants, and $\mathrm{k}_{1}$ and $\mathrm{k}_{2}$ are the fast and slow decay rates, respectively. Notably, the strength of the Raman signal itself is not seen to evolve over the duration of exposure. 


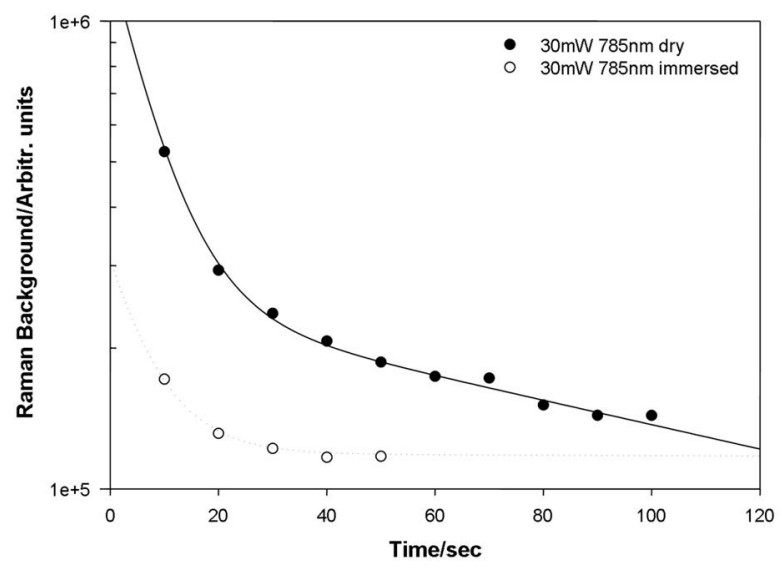

Fig.3: Representation decay of the background at $\sim 1750 \mathrm{~cm}^{-1}$ over time in the Raman spectra recorded from human skin using a $785 \mathrm{~nm}$ laser source.

It has recently been reported that working with immersed tissue sections results in significantly reduced spectral background and improved quality spectra [33]. Therefore, the skin sections were immersed in water for $60 \mathrm{mins}$ before and during recording in order to visualise the effect on the evolution of the background over time. The accumulation time was kept at $10 \mathrm{~s}$ and the laser power at $30 \mathrm{~mW}$ to allow comparison between the data sets. The first spectrum obtained for the immersed skin section is presented in the figure $2 \mathrm{~B}$ (top spectrum). The definition of the peaks has been greatly improved and the background is significantly reduced compared to the spectrum recorded on the dry skin, as previously reported [33]. The features are largely the same as those observed in the dry state, although slight variations of the relative intensities of bands are evident, at for example $900-950 \mathrm{~cm}^{-1}$, and $\sim 1050 \mathrm{~cm}^{-1}$. These variations may be due to point to point variability of the spectra, or due to slight changes in morphology from the dry to immersed state. As shown in figure 3, the background evolves only slightly over the first 30 seconds exposure, at the same rate as the dry tissue section $\left(\mathrm{k}_{1}=0.13 \mathrm{sec}^{-1}\right)$, but after this time the background has stabilised and no further evolution is observable 
$\left(\mathrm{k}_{2}<0.001 \mathrm{sec}^{-1}\right.$ compared to $\mathrm{k}_{2}=0.006 \mathrm{sec}^{-1}$ for dry tissue sections). At a time point of 60 sec, the background has reduced to close to that of the objective immersed in water, focussed on a $\mathrm{CaF}_{2}$ substrate in the high wavenumber regime $\left(\sim 1750 \mathrm{~cm}^{-1}\right)$ although it remains substantially higher in the low wavenumber regime $\left(400 \mathrm{~cm}^{-1}\right)$ (data not shown). Again, no physical degradation of the sample is visibly discernible after prolonged exposure.

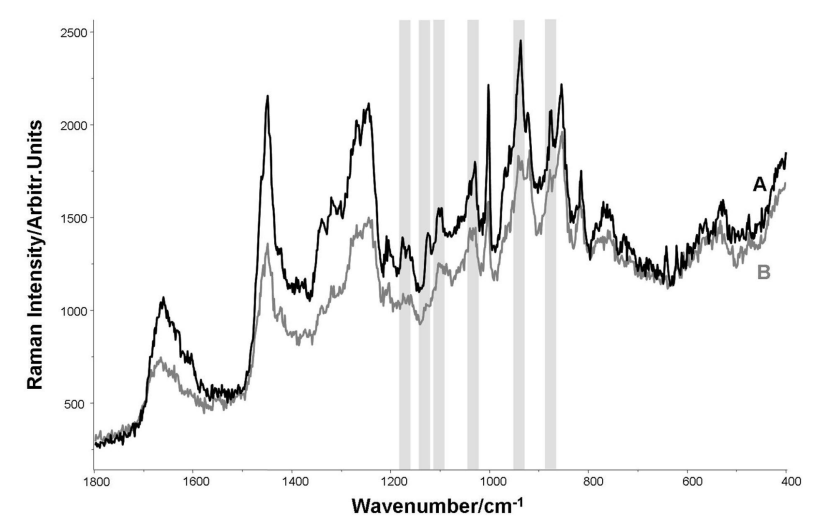

Fig.4: Comparison of the Raman spectra collected from human skin using a $785 \mathrm{~nm}$ laser source from dry and immersed sections. Spectrum $A$ has been recorded from immersed section and corresponds to $1 \times 10$ s acquisition. Spectrum $B$ has been obtained from a dry section and corresponds to the last of $10 \times 10$ s acquisitions. The spectra have been corrected for $\mathrm{CaF}_{2}$ and $\mathrm{CaF}_{2}+$ water for comparison of the signals and peaks intensities.

The spectrum of the $10^{\text {th }}$ acquisition of $10 \mathrm{~s}$ recorded from the dry sample is compared with the spectrum collected in immersion after $1 \times 10$ s acquisition in figure 4A and 4B. The spectra exhibit similar backgrounds after correction for $\mathrm{CaF}_{2}$ and water contributions. Although the spectra have similar intensities in the range $400-850 \mathrm{~cm}^{-1}$, the relative amplitudes of the bands between $900-1800 \mathrm{~cm}^{-1}$ are increased in the case of measurements performed on immersed tissues, highlighting the increase in signal to background ratio. 
In addition to the overall improved definition of the features in the immersion spectrum, the feature at $\sim 850 \mathrm{~cm}^{-1}$, corresponding to $v(\mathrm{C}-\mathrm{C})$ of the hydroxy-proline ring, is significantly more pronounced in immersion (figure 4A). The peaks at $938 \mathrm{~cm}^{-1}(v(\mathrm{C}-\mathrm{C})$ of protein backbone), $1030 \mathrm{~cm}^{-1}$ (CH plane bending for phenylalanine), $1099 \mathrm{~cm}^{-1}(\mathrm{v}(\mathrm{C}-$ $\mathrm{N})$ ), $1126 \mathrm{~cm}^{-1}$ (protein $\mathrm{C}-\mathrm{N}$ stretch) and $1177 \mathrm{~cm}^{-1}\left(\mathrm{NH}_{3}\right)$ also exhibit modified intensities in the immersion measurements [40, 41]. As the spectral features do not evolve significantly as a function of exposure, these spectral differences are simply attributable to slight changes in the morphology in the hydrated state of the tissue compared to the dry state.

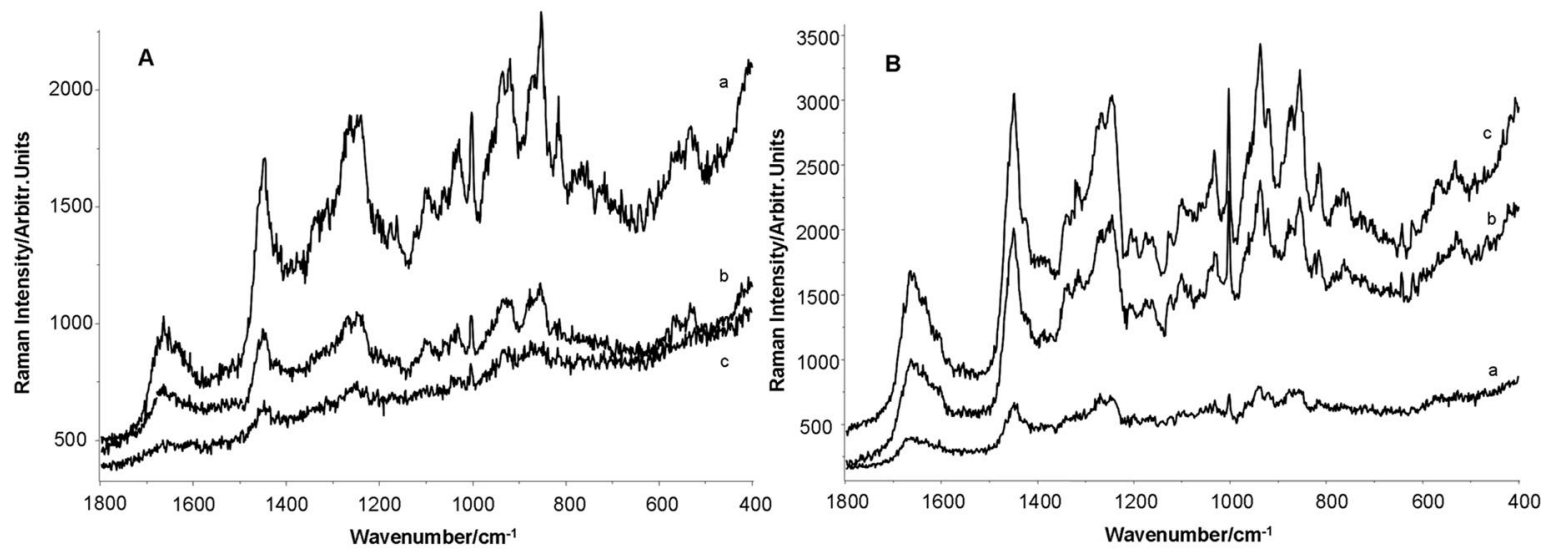

Fig.5: Comparison of Raman spectra recorded on human skin (A) dry and (B) immersed for different $785 \mathrm{~nm}$ laser power settings: (a) $5 \mathrm{~mW}$, (b) $15 \mathrm{~mW}$ and (c) $30 \mathrm{~mW}$. The spectra recorded from the dry skin are those recorded after 10x10s acquisitions whereas for the immersed skin they correspond to $1 \times 10$ s acquisitions.

The laser power also influences the profile of the spectra recorded. Similar measurements were performed on the dry skin with a laser power of $15 \mathrm{~mW}$ and $5 \mathrm{mw}$ (figure $5 \mathrm{Ab}$ and figure 5Aa respectively). When using $5 \mathrm{~mW}$, the spectra obtained after extended exposure (10x10s) exhibit a poor signal to background ratio and no specific features can 
be identified even after extended exposure to the laser. An intensity of at least $15 \mathrm{~mW}$ is needed to be able to collect Raman spectra containing useful information, although the spectral quality is significantly improved at $30 \mathrm{~mW}$ (figure 5Ac). When working using immersed sections, the laser power has less influence on the data recorded and with a laser power of $15 \mathrm{~mW}$ the spectra are comparable to those obtained with $30 \mathrm{~mW}$ (figure $5 \mathrm{Bb}$ and $5 \mathrm{Bc}$ respectively). The spectra recorded using $5 \mathrm{~mW}$ (figure $5 \mathrm{Ba}$ ) exhibit identifiable peaks but the signal to background ratio remains quite poor for specific analysis of the different features present.

Laser power and exposure time each have an effect on the background present in the spectra recorded, as well as the signal to noise ratio. Working using immersion greatly improves the quality of the data, enhancing considerably the definition of the peaks present in the spectra. The combination of high laser power and immersion appears to be optimal for tissue sections at $785 \mathrm{~nm}$. The signal to background present in the first spectrum recorded after $10 \mathrm{~s}$ is similar to the spectrum recorded from dry skin after 10 acquisitions of 10s. Moreover, after 20s, the spectra recorded exhibit stable intensities and no further improvement can be seen. Thus, for the purpose of spectral mapping, the immersed tissue would need a 10s pre-exposure at each point (usually described as a photobleaching period in the acquisition software) before starting the recording of spectra, instead of almost 100s for the dry tissue, considerably shortening the time required for the mapping of the area selected. This provides the possibility to increase the number of spectra contained in a map, yielding higher spatial resolution or larger scan areas, or simply a more rapid screening process. 


\subsection{Presence of a background in the spectra recorded using the $660 \mathrm{~nm}$ source}

The response of the skin sections to the $660 \mathrm{~nm}$ laser line was then tested in a similar fashion. In the previous section, it was demonstrated that the laser power has an impact on the intensity of the background present in the Raman spectra. For this reason, the laser source was set to the maximum available power, $15 \mathrm{~mW}$ at the objective. Firstly, the dry skin was measured with 10 s accumulations repeated successively. Figure $6 \mathrm{~A}$ presents the different spectra obtained, the top spectrum being the first recorded. As observed for the $785 \mathrm{~nm}$ source, the background is initially strong, making the identification of the different peaks present in the spectrum quite difficult. With extended exposure to the laser source, however, the overall intensity of the Raman spectrum decreases, associated with a diminution of the background. As was the case for prolonged exposure to the $785 \mathrm{~nm}$ source, no physical degradation of the sample is visibly discernible after prolonged exposure. At $660 \mathrm{~nm}$, immersed skin sections exhibit a similar behaviour to that observed with $785 \mathrm{~nm}$ as source and the background decreases only slightly over time (figure 6B). Figure 7 compares the time evolution of the background at $\sim 1750 \mathrm{~cm}^{-1}$ at $15 \mathrm{~mW}$ in the dry and immersed states. As was the case for $785 \mathrm{~nm}$, the evolution is best fit with a double exponential behaviour. The initial rapid evolution occurs at a rate of $\mathrm{k}_{1}=$ $0.12 \mathrm{sec}^{-1}$, while the slower decay occurs at a rate of $\mathrm{k}_{2}=0.007 \mathrm{sec}^{-1}$. In immersion, a similar initial decay of $\mathrm{k}_{1}=0.12 \mathrm{sec}^{-1}$ is observed, but no secondary decay is discernible $\left(\mathrm{k}_{2}<0.001 \mathrm{sec}^{-1}\right)$. The time evolution, within the resolution of the experiment, is thus almost identical to that observed at $785 \mathrm{~nm}$, suggesting a similar physical origin. 

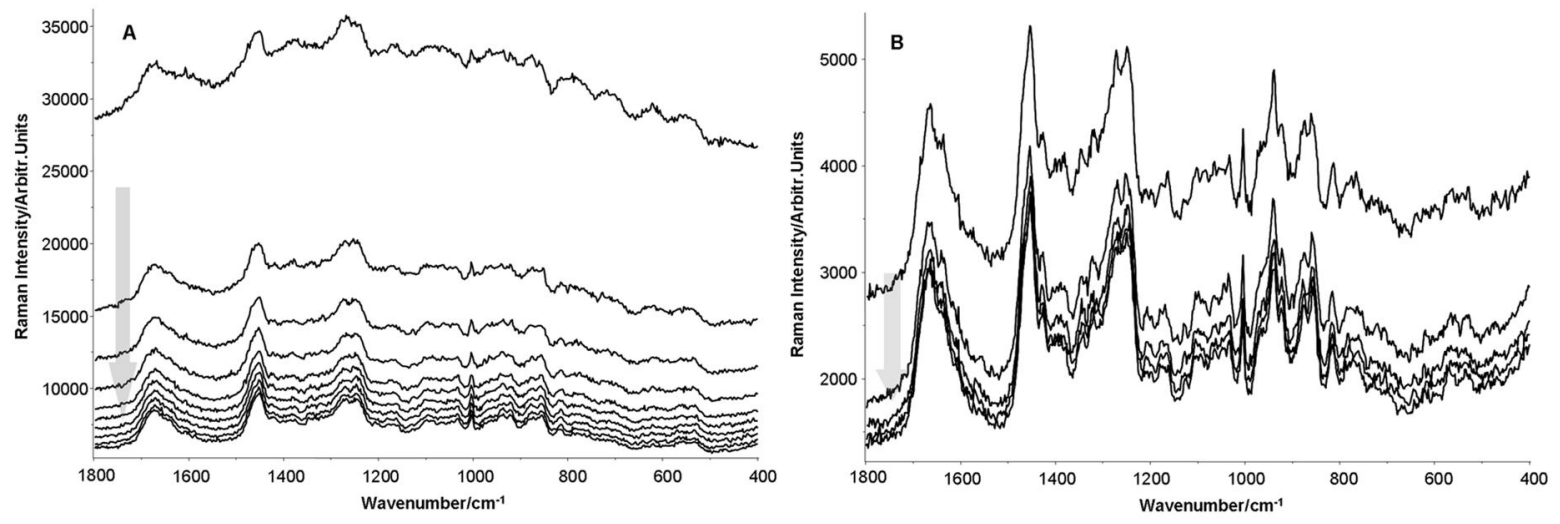

Fig.6: Raman spectra recorded from human skin sections placed on $\mathrm{CaF}_{2}$ windows. All spectra have been recorded using the $660 \mathrm{~nm}$ wavelength delivering $15 \mathrm{~mW}$ at the objective with an acquisition time of 10s. Repeated measurements have been performed every 10s on dry (A) and immersed (B) skin. No pre-processing has been performed and no offset has been added to the plot.

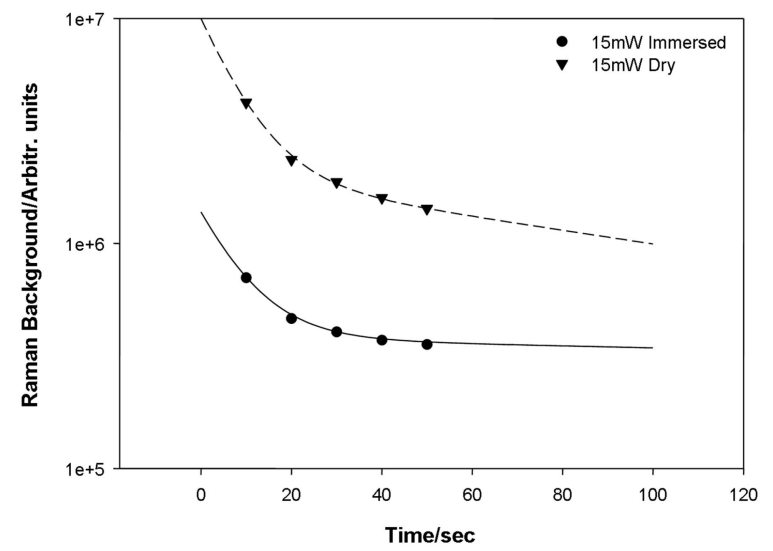

Fig.7: Representation of the background decay over time in the Raman spectra recorded from the human skin using a $660 \mathrm{~nm}$ laser source. 


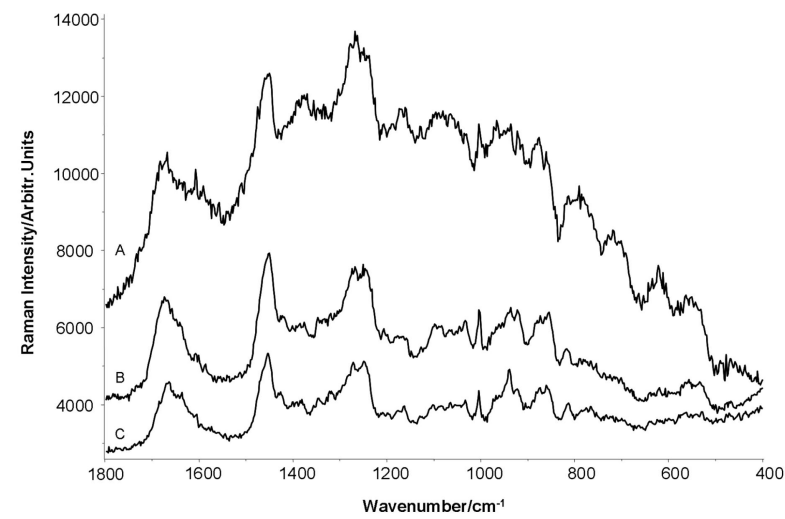

Fig.8: Comparison of Raman spectra of human skin recorded using the $660 \mathrm{~nm}$ on a dry section after $1 \times 10$ s acquisition (A), and after 10x10s acquisition (B) and an immersed section after $1 \times 10$ s acquisition (C). For clarity, spectrum (A) has been decreased by a factor of 3 .

For clarity, figure 8 displays the spectrum recorded from the dry skin after 10s (figure $8 \mathrm{~A}$ ) compared with the spectrum obtained for the $10^{\text {th }} 10 \mathrm{~s}$ acquisition (figure $8 \mathrm{~B}$ ). The diminution of the background intensity is clear for extended exposure to the laser source. The first spectrum also appears to be distorted in the region $600-1600 \mathrm{~cm}^{-1}$. After $100 \mathrm{~s}$ exposure to the laser in the dry state, the Raman spectra collected exhibit a significantly diminished background and the peaks in the spectral window $700-1100 \mathrm{~cm}^{-1}$ are clearly visible. The spectrum of figure $8 \mathrm{C}$ was recorded on the immersed skin using $1 \mathrm{x} 10 \mathrm{~s}$ accumulation. The background is comparable with that of the spectrum in figure $8 \mathrm{~B}$ and the information contained appears identical to the observations at $785 \mathrm{~nm}$ (figure 4). Notably, all the features which were identified as enhanced in immersion at $785 \mathrm{~nm}$ are also enhanced in immersion at $660 \mathrm{~nm}$ and the enhancement is attributed to a change in tissue morphology in the hydrated state. Overall, the observations demonstrate the ease of 
collection of Raman spectra using shorter exposure time when working with immersed tissue sections.

\subsection{Benefits of immersed sections for $532 \mathrm{~nm}$ and $473 \mathrm{~nm}$ wavelengths}

The $532 \mathrm{~nm}$ and $473 \mathrm{~nm}$ laser sources available for the measurement can deliver up to 30 $\mathrm{mW}$ at the objective and, given the intrinsic dependence of the Raman scattering phenomenon on source wavelength [42], have the advantage of delivering relatively stronger spectra in relatively short acquisition times. Nevertheless, their use for biological samples can be limited due to the presence of a background in the spectra recorded often associated to fluorescence in the literature $[23,24,43]$ or the photo-degradation of the samples under investigation [39]. Both effects have significant impact on the quality and reliability of the recordings, bringing into question the suitability of these laser lines with tissue sample analysis.

Photo-degradation, clearly visible on the video image as the apparition of a black spot where the measurement has been performed (not shown), limits the intensity of the laser which can be used. The photo-degradation is abrupt and results in the detector being swamped by background. In the case of dry skin, using the x100 objective and $10 \mathrm{~s}$ acquisition, $5 \mathrm{~mW}$ is the maximum laser power that could possibly be used for both the $532 \mathrm{~nm}$ and $473 \mathrm{~nm}$ sources. An increase of the laser power results in sample damage before recording of the first spectrum. At this power, the signal to background/noise ratio for $10 \mathrm{~s}$ acquisitions was too poor to visualise any information in the spectra recorded (data not shown) and therefore the potential to investigate the skin samples using these laser lines seemed to be compromised. Moreover, even extended exposure to the laser at 
$5 \mathrm{~mW}$ up to 200s did not result in a Raman spectrum with identifiable features from the dry skin.

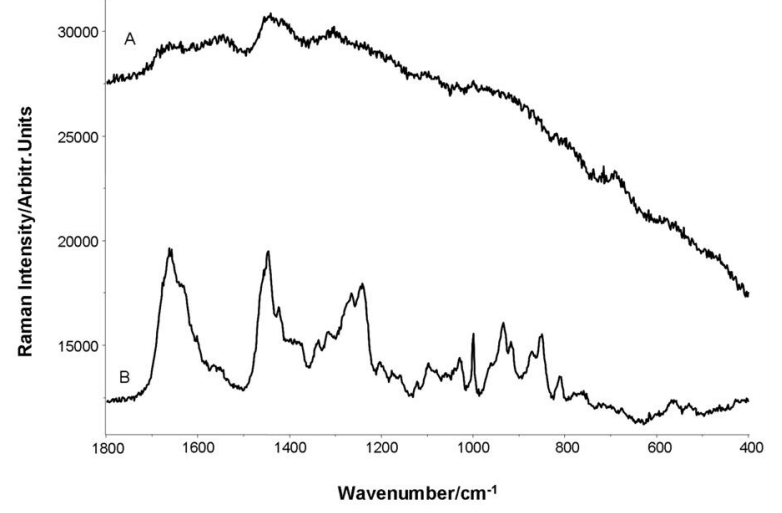

Fig.9: Raman spectra collected from human skin using the $532 \mathrm{~nm}$ wavelength. Spectrum (A) has been obtained from a dry section using a laser power of $5 \mathrm{~mW}$ and results from a $1 \times 10$ s acquisition. Spectrum (B) has been recorded from an immersed section using a 30 $m W$ laser power and results from a $1 \times 10$ s acquisition.

However, immersion of the skin sections in water during the measurements was found to protect the samples from photo-degradation. Using the immersion set up, the laser power could be increased to $30 \mathrm{~mW}$ without any obvious degradation of the skin samples. When working on dry skin, the limited laser power employable restricts the possibility to collect spectra containing exploitable information (figure 9A). Working in immersion, it is possible to record spectra exhibiting all the different features characteristic of the skin even after 1x10s exposure (figure 9B). Although the spectrum recorded after short time exposure contains all the specific feature of the skin, as was the case for the $785 \mathrm{~nm}$ and $660 \mathrm{~nm}$ laser lines, the overall Raman intensity decreases over time, indicating a decrease of the background. After 5 acquisitions of $10 \mathrm{~s}$, the signal is stabilised and no further improvement is observed (figure 10). The time evolution of the background to the Raman 
spectra recorded at $532 \mathrm{~nm}, 30 \mathrm{~mW}$ in immersion is shown in Figure 11. Similar to the case of $785 \mathrm{~nm}$ and $660 \mathrm{~nm}$, a relatively rapid initial evolution is observed, with a rate constant of $\mathrm{k}_{1}=0.016 \mathrm{sec}^{-1}$ after which a slow or negligible evolution is observed $\left(\mathrm{k}_{2}\right.$ $\left.<0.003 \mathrm{sec}^{-1}\right)$.

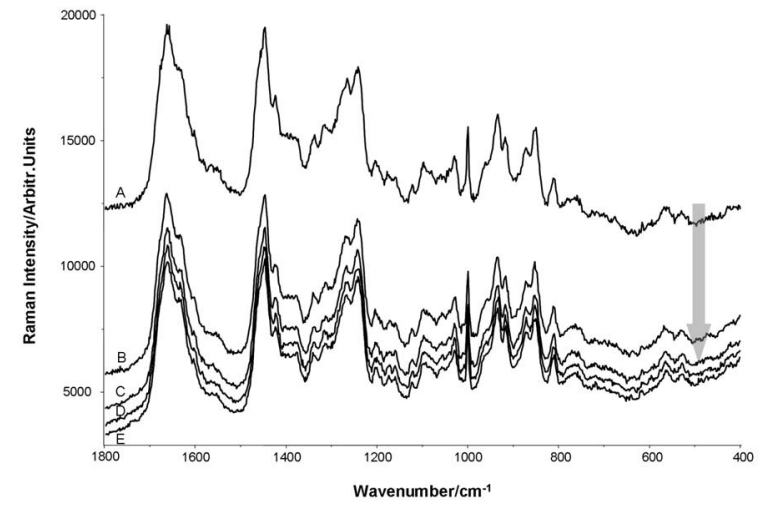

Fig.10: Raman Spectra collected from an immersed human skin section using the $532 \mathrm{~nm}$ wavelength at $30 \mathrm{~mW}$. Spectra result from $1 \times 10$ s acquisitions and have been recorded sequentially at the same spot.

Similar observations have been made with the $473 \mathrm{~nm}$ wavelength. The recording of Raman spectra from the dry skin was limited to the use of $5 \mathrm{~mW}$ laser power. However, using immersed skin sections, a $30 \mathrm{~mW}$ laser power allowed the collection of Raman spectra with a greatly reduced background after short exposure times (figure 12). As shown in Figure 11, the initial decay has a similar rate, $\mathrm{k}_{1}=0.16 \mathrm{sec}^{-1}$, but in contrast to the behaviour observed for all other laser lines in immersion, a significant decay persists after this time, at a rate of $\mathrm{k}_{2}=0.011 \mathrm{sec}^{-1}$. No damage to the skin was observed and a minimum background can be seen in the spectra after 50 s exposure to the laser source. 


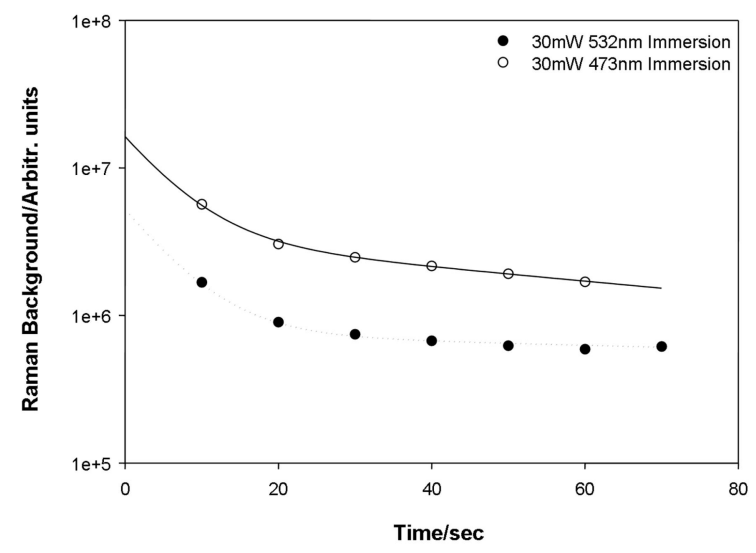

Fig. 11: Representation of the background decay over time in the Raman spectra recorded from the human skin using $532 \mathrm{~nm}$ and $473 \mathrm{~nm}$ laser sources.

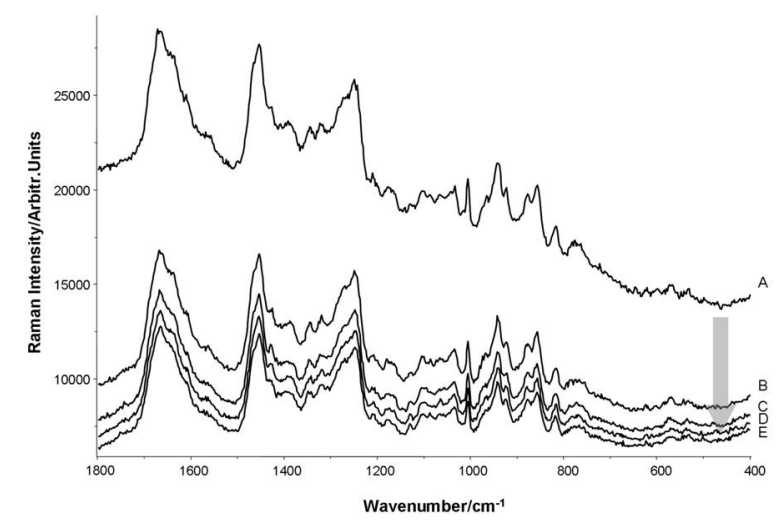

Fig. 12: Raman Spectra collected from an immersed human skin section using the $473 \mathrm{~nm}$ wavelength at $30 \mathrm{~mW}$. Spectra result from $1 \times 10$ s acquisitions and have been recorded sequentially at the same spot.

\subsection{Discussion}

The dermis is a relatively thick layer of the skin, varying between $0.6 \mathrm{~mm}$ on the eyelids up to $3 \mathrm{~mm}$ on the palms and soles. It can be subdivided in two different layers, the papillary and reticular layer. The predominant cellular type that can be found in the dermis is fibroblasts, producing collagen, elastin and structural proteoglycans conferring 
strength and toughness to the skin without loss of the elasticity and viscosity of the tissue [44]. However, the collagen can represent up to $75 \%$ of the dry weight and $90 \%$ of the total protein content which results in the strong similarity between Raman spectra collected from the skin and the spectrum of pure collagen [30, 40, 45]. In addition, the dermis also contains a number of chromophores such as keratin, NAD/NADH, bilirubin, and porphyrins. Of the molecular components of the dermis, only elastin $\left(\lambda_{\mathrm{abs}}=\right.$ $\left.440 \mathrm{~nm} / 460 \mathrm{~nm}, \lambda_{\mathrm{em}}=500 \mathrm{~nm} / 540 \mathrm{~nm}\right)$, keratins $\left(\lambda_{\mathrm{abs}}=370 \mathrm{~nm}, \lambda_{\mathrm{em}}=460 \mathrm{~nm}\right)$, $\mathrm{NAD} / \mathrm{NADH}\left(\lambda_{\mathrm{abs}}=350 \mathrm{~nm}, \lambda_{\mathrm{em}}=460 \mathrm{~nm}\right)$ and porphyrins $\left(\lambda_{\mathrm{abs}}=405 \mathrm{~nm}, \lambda_{\mathrm{em}}=600 \mathrm{~nm}\right)$ are reported to exhibit fluorescence in the visible region [25]. Notably, melanin, which is resonant and potentially fluorescent at wavelengths as long as $785 \mathrm{~nm}$, is concentrated in the stratum basale layer, the innermost layer of the epidermis which lies adjacent to the dermis [30]. Of the other molecular components identified above, the most likely candidate to be resonant and contribute fluorescence to the Raman background is elastin at $473 \mathrm{~nm}$. Notably, however, the absorption maxima in all cases are far from the wavelengths of the other sources utilised in these studies, and although red shifts of absorption features are to be expected in the dried state, it can be expected that even $532 \mathrm{~nm}$ is sufficiently distant from the resonances of the identifiable chromophores.

At $785 \mathrm{~nm}$, the intensities employed are too low for two photon excitation, a nonlinear optical process normally requiring pulsed excitation intensities and short durations, and the background to the Raman spectrum is thus unlikely to have origin in fluorescence. In pure protein samples, it has been demonstrated that at this wavelength the Raman background depends on sample morphology, and that in tissue sections the background is essentially eliminated by immersion [33]. In the protein rich and physically 
inhomogeneous dermis, the background is thus attributed to scattering of the laser source and even the Raman peaks themselves, and the reduction in immersion is attributed to improved refractive index matching between the water and the tissue, compared to the air/tissue interface. Thus the evolution of the background is attributed to photothermal "annealing" like effects rather than photochemical bleaching, which, although not visible microscopically, result in local changes to sample morphology and a reduction in scattering. Photothermal annealing is a commonly employed technique in for example semiconductor thin film structural processing [46]. Using the 473 and $532 \mathrm{~nm}$ lasers on dry skin, the recording of spectra is impossible, as the sample is visibly damaged, even at reduced laser powers as low as $5 \mathrm{~mW}$. When working in immersion, the damage to the tissue does not occur, even under higher power illumination. This effect is most likely due to the improved thermal heat sinking of the tissue in the immersed environment. The origin of the two stage process in the dry state is not clear, however. In immersion, the initial rapid decay of the background is also observed, albeit weakly compared to the dry sections. However, the signal is seen to stabilise after approximately ten seconds, and no secondary slow decay is observable. It is assumed therefore that the improved heat sinking environment of the water environment reduces and eliminates the secondary decay.

A similar behaviour is observable at $660 \mathrm{~nm}$ and even $532 \mathrm{~nm}$, and this might be expected, as there are no identifiable fluorophores which are excited in these spectral regions. Notably, for $532 \mathrm{~nm}$, the thermal heat sink of the water environment significantly reduces the risk of photodamage, enabling measurement at elevated powers. At $473 \mathrm{~nm}$ however, elastin has a significant absorption and fluorescence which potentially contributes to the 
Raman background. Notably, for the same measurement conditions, the background at $473 \mathrm{~nm}$ is considerably higher than that observed at $532 \mathrm{~nm}$ and a significant secondary decay is observable even in immersion. This is potentially attributable to the contribution of fluorescence from, and photo-bleaching of, the elastin fluorophore.

\section{Conclusion}

Whereas dried tissue sections exhibit a strong background to the Raman spectrum at all source wavelengths, measurement in water immersion significantly reduces or eliminates this background. The water environment provides an index matching environment which reduces stray light due to scatter, and also a thermal heat sink to reduce photothermal damage. The spectral definition is furthermore improved, and several features are enhanced in the hydrated tissue.

At all but the $473 \mathrm{~nm}$ wavelength, no potentially contributing fluorophores are identifiable. At this wavelength, the background is relatively higher and is attributed to fluorescence from elastin. The secondary temporal decay persists, independent of the environment, indicating a photochemical process. Thus it is concluded that fluorescence is only a significant contributor to the background at short source wavelengths.

Overall, measurement in immersion greatly improves spectral quality and sample stability and reduces spectral background. Measurements at elevated powers are enabled across the visible spectrum with no photothermal damage. In addition to greatly reducing acquisition times for mapping, preprocessing requirements are reduced to removal of 
water and any substrate contributions, greatly enhancing the reliability of spectra for diagnostic applications.

\section{Acknowledgements}

This research was supported by the National Biophotonics and Imaging Platform (NBIP) Ireland funded under the Higher Education Authority PRTLI (Programme for Research in Third Level Institutions) Cycle 4, co-funded by the Irish Government and the European Union Structural Fund. 


\section{REFERENCES}

[1] F. Bonnier, D. Bertrand, S. Rubin, L. Venteo, M. Pluot, B. Baehrel, M. Manfait, G.D. Sockalingum, Analyst 133 (2008) 784.

[2] M. Larraona-Puy, A. Ghita, A. Zoladek, W. Perkins, S. Varma, I.H. Leach, A.A. Koloydenko, H. Williams, I. Notingher, J Biomed Opt 14 (2009) 054031.

[3] F.M. Lyng, E.O. Faolain, J. Conroy, A.D. Meade, P. Knief, B. Duffy, M.B.

Hunter, J.M. Byrne, P. Kelehan, H.J. Byrne, Exp Mol Pathol 82 (2007) 121.

[4] A. Tfayli, O. Piot, A. Durlach, P. Bernard, M. Manfait, Biochim Biophys Acta 1724 (2005) 262.

[5] D. Moss. 2011. Biomedical Applications of Synchrotron Infrared Microspectroscopy: A practical Approach. Cambridge: RCS Publishing.

[6] A.D. Meade, H.J. Byrne, F.M. Lyng, Mutat Res 704 (2010) 108.

[7] P. Knief, C. Clarke, E. Herzog, M. Davoren, F.M. Lyng, A.D. Meade, H.J. Byrne, Analyst 134 (2009) 1182.

[8] J. Ling, S.D. Weitman, M.A. Miller, R.V. Moore, A.C. Bovik, Appl Opt 41 (2002) 6006.

[9] D. Sebiskveradze, V. Vrabie, C. Gobinet, A. Durlach, P. Bernard, E. Ly, M. Manfait, P. Jeannesson, O. Piot, Lab Invest 91 (2011) 799.

[10] R. Wolthuis, A. Travo, C. Nicolet, A. Neuville, M.P. Gaub, D. Guenot, E. Ly, M. Manfait, P. Jeannesson, O. Piot, Anal Chem 80 (2008) 8461.

[11] F. Draux, P. Jeannesson, C. Gobinet, J. Sule-Suso, J. Pijanka, C. Sandt, P. Dumas, M. Manfait, G.D. Sockalingum, Anal Bioanal Chem 395 (2009) 2293.

[12] H. Nawaz, F. Bonnier, P. Knief, O. Howe, F.M. Lyng, A.D. Meade, H.J. Byrne, Analyst 135 (2010) 3070.

[13] F. Bonnier, P. Knief, B. Lim, A.D. Meade, J. Dorney, K. Bhattacharya, F.M. Lyng, H.J. Byrne, Analyst 135 (2010) 3169.

[14] M. Miljkovic, T. Chernenko, M.J. Romeo, B. Bird, C. Matthaus, M. Diem, Analyst 135 (2010) 2002.

[15] C. Krafft, D. Codrich, G. Pelizzo, V. Sergo, J Biophotonics 1 (2008) 154.

[16] C. Krafft, D. Codrich, G. Pelizzo, V. Sergo, Analyst 133 (2008) 361.

[17] S. Venyaminov, F.G. Prendergast, Analytical biochemistry 248 (1997) 234.

[18] F. Bonnier, A.D. Meade, S. Merzha, P. Knief, K. Bhattacharya, F.M. Lyng, H.J. Byrne, Analyst 135 (2010) 1697.

[19] F. Draux, P. Jeannesson, A. Beljebbar, A. Tfayli, N. Fourre, M. Manfait, J. SuleSuso, G.D. Sockalingum, Analyst 134 (2009) 542.

[20] P. Bassan, H.J. Byrne, F. Bonnier, J. Lee, P. Dumas, P. Gardner, Analyst 134 (2009) 1586.

[21] P. Bassan, H.J. Byrne, J. Lee, F. Bonnier, C. Clarke, P. Dumas, E. Gazi, M.D. Brown, N.W. Clarke, P. Gardner, Analyst 134 (2009) 1171.

[22] P. Bassan, A. Kohler, H. Martens, J. Lee, H.J. Byrne, P. Dumas, E. Gazi, M. Brown, N. Clarke, P. Gardner, Analyst 135 (2010) 268.

[23] C.A. Lieber, A. Mahadevan-Jansen, Applied spectroscopy 57 (2003) 1363.

[24] J. Zhao, H. Lui, D.I. McLean, H. Zeng, Applied spectroscopy 61 (2007) 1225.

[25] N. Kollias, R. Gillies, M. Moran, I.E. Kochevar, R.R. Anderson, J Invest

Dermatol 111 (1998) 776. 
[27] E.B. Hanlon, R. Manoharan, T.W. Koo, K.E. Shafer, J.T. Motz, M. Fitzmaurice, J.R. Kramer, I. Itzkan, R.R. Dasari, M.S. Feld, Phys Med Biol 45 (2000) R1.

[28] Z. Huang, H. Lui, X.K. Chen, A. Alajlan, D.I. McLean, H. Zeng, J Biomed Opt 9 (2004) 1198.

[29] Z. Huang, H. Zeng, I. Hamzavi, A. Alajlan, E. Tan, D.I. McLean, H. Lui, J Biomed Opt 11 (2006) 34010.

[30] G.F. Odland. 1991. Structure of the skin. in L. A. Goldsmith, ed. Physiology, Biochemistry and molecular Biology of the skin. New York: Oxford University Press.

[31] J.P. Biscar, N. Kollias, Chem. Phys. Lett. 24 (1974) 563.

[32] J.L. Koenig, B. Frushour, Biopolymers 11 (1972) 1871.

[33] F. Bonnier, S.M. Ali, P. Knief, A.D. Meade, W. Hornebeck, H. Lambkin, K. Flynn, V. McDonagh, C. Healy, T.C. Lee, F.M. Lyng, H.J. Byrne, Journal of Raman spectroscopy DOI 10.1002/jrs.2825 (2010).

[34] B.D. Beier, A.J. Berger, Analyst 134 (2009) 1198.

[35] S.C. Zhi-Min Zhang, Yi-Zeng Liang, Zhao-Xia Liu, Qi-Ming Zhang, Li-Xia Ding, Fei Ye and Hua Zhou, Journal of Raman spectroscopy 41 (2010) 659.

[36] G. Shetty, C. Kendall, N. Shepherd, N. Stone, H. Barr, Br J Cancer 94 (2006) 1460.

[37] G.J. Puppels, F.F. de Mul, C. Otto, J. Greve, M. Robert-Nicoud, D.J. Arndt-Jovin, T.M. Jovin, Nature 347 (1990) 301.

[38] G.J. Puppels, H.S. Garritsen, G.M. Segers-Nolten, F.F. de Mul, J. Greve, Biophys J 60 (1991) 1046.

[39] G.J. Puppels, J.H. Olminkhof, G.M. Segers-Nolten, C. Otto, F.F. de Mul, J. Greve, Experimental cell research 195 (1991) 361.

[40] S. Jaisson, S. Lorimier, S. Ricard-Blum, G.D. Sockalingum, C. Delevallee-Forte, G. Kegelaer, M. Manfait, R. Garnotel, P. Gillery, Chem Biol 13 (2006) 149.

[41] A. Tfayli, O. Piot, F. Draux, F. Pitre, M. Manfait, Biopolymers 87 (2007) 261.

[42] A.L. Derek. 2002. The Raman Effect: A Unified Treatment of the Theory of Raman Scattering by Molecules. New York: John Wiley \& Sons Ltd.

[43] V. Mazet, C. Carteret, D. Brie, J. Idier, B. Humbert, Chemometrics and Intelligent Laboratory Systems 76 (2005) 121.

[44] J.A. MacGrath, R.A.J. Eady, F.M. Pope. 2004. Anatomy and organization of human skin. In: Rook's Textbook of Dermatology. Oxford Blackwell.

[45] P.J. Caspers, G.W. Lucassen, R. Wolthuis, H.A. Bruining, G.J. Puppels, Biospectroscopy 4 (1998) S31.

[46] S. Jia, H. Ge, X. Geng, W. Z., Solar Energy Materials and Solar Cells Volume 62 (2000) 201. 